\title{
Randomized clinical trial comparing LigaSure haemorrhoidectomy with open diathermy haemorrhoidectomy
}

\author{
K.-Y. Tan • T. Zin • H.-L. Sim • P.-L. Poon • \\ A. Cheng • K. Mak
}

Received: 7 December 2007 / Accepted: 28 March 2008 / Published online: 9 June 2008

(c) Springer-Verlag 2008

\begin{abstract}
Background Milligan-Morgan excision haemorrhoidectomy remains a very popular treatment modality for third and fourth degree haemorrhoids due to its cost effectiveness and good long-term results. The LigaSure tissue-sealing device is an alternative technique used in haemorrhoidectomy that has been shown to produce favourable results. The aim of this study was to assess the effectiveness of the LigaSure tissue sealing device in comparison with conventional diathermy haemorrhoidectomy. Methods A prospective clinical trial was conducted. Patients with newly diagnosed haemorrhoids requiring haemorrhoidectomy were randomized to either LigaSure haemorrhoidectomy or diathermy haemorrhoidectomy. Surgical technique and postoperative care was standardized. Outcome measures were operative time and bleeding, postoperative pain (measured on a visual analogue scale) and rate of wound healing. Results We randomized 44 patients, 22 to LigaSure and 22 to diathermy; 43 patients were evaluated. They were aged between 19 and 71 years. There were no differences in patient demographics or type of haemorrhoid being oper-
\end{abstract}

K.-Y. Tan (四) · T. Zin · H.-L. Sim · P.-L. Poon · A. Cheng $\cdot$ K. Mak Department of Surgery

Colorectal Service

Alexandra Hospital

378 Alexandra Road, Singapore 159964

E-mail: kokyangtan@gmail.com ated on. LigaSure haemorrhoidectomy had a significantly lower mean operative time and intraoperative bleeding. At 3 weeks after surgery, haemorrhoidectomy performed with LigaSure had an odds ratio for complete epithelialization of 3.1 over diathermy (95\% CI 1.2-8.2). There was no difference in postoperative pain. Conclusions LigaSure haemorrhoidectomy is superior to diathermy for open haemorrhoidectomy.

Keywords LigaSure · Haemorrhoidectomy · Wound healing · Pain

\section{Introduction}

Despite the current interest in stapled haemorrhoidopexy, conventional Milligan-Morgan open haemorrhoidectomy remains a frequently performed operation in our country. The lower cost of the procedure associated with a good long-term cosmetic effect in patients with large skin tags make open haemorrhoidectomy a viable option still. An Italian study published very recently actually found the Milligan-Morgan procedure to have better results than stapled haemorrhoidopexy [1]. These results were echoed in a systematic review a few months previously [2].

The use of the LigaSure tissue-sealing device provides an alternative to performing open haemorrhoidectomy for third and fourth degree piles. Some Western studies have shown benefits of using LigaSure over diathermy in terms of operative time, postoperative pain and wound healing [3-5].

The aim of this study was to assess the effectiveness of the LigaSure tissue sealing device in comparison with conventional diathermy haemorrhoidectomy in our setting and population. We sought to assess LigaSure in 
terms of ease of use, postoperative pain and wound healing rate at our centre.

\section{Patients and methods}

A prospective randomized clinical trial was conducted at our centre. All patients with newly diagnosed symptomatic third and fourth degree haemorrhoids requiring haemorrhoidectomy were included in the recruitment for the trial. For inclusion patients had to be between 21 to 80 years of age. This trial was investigator-initiated. The distributor of the LigaSure tissue-sealing system provided the electrodes for the trial so that the extra cost of treatment with LigaSure would not be passed on to the patients.

Patients were excluded if they were undergoing a combined procedure for fissures or fistulae, if they were allergic to any of the standard medications prescribed for the trial, or if they did not consent to the trial.

\section{Study design}

All patients were randomized to receive either LigaSure haemorrhoidectomy or conventional haemorrhoidectomy using sealed envelopes. The randomization performed was nonconsecutive in nature. The envelopes were sequentially numbered with allocations determined before the trial commenced. Randomization was performed in the operating theatre just prior to surgery. A standard medication package was issued to all patients postoperatively. Patients were blinded to the type of surgery performed. The National Healthcare Group of Singapore Domain Specific Review Board endorsed this trial.

\section{Surgery}

A standardized general anaesthetic technique using propofol, fentanyl and inhalation anaesthesia was used. All patients were given a Fleet enema prior to surgery. Patients underwent Milligan-Morgan open haemorrhoidectomy using either LigaSure or diathermy according to the randomization. In all patients $10 \mathrm{ml} 0.25 \%$ bupivacaine was injected into the perianal skin prior to dissection. The pile masses were retracted and dissected off the internal sphincter using either diathermy or the LigaSure vesselsealing device. The pedicles were secured clear of the internal sphincter and the resected wound left open to granulate with adequate skin bridges. The operative time was recorded by an operating theatre nurse. Blood loss was recorded as the number of soaked gauzes.
Postoperative care

All patients were prescribed Fybogel (one sachet daily for 2 weeks) to aid in defecation after the operation. All patients were prescribed Daflon two tablets twice daily for 2 weeks. Paracetamol $1 \mathrm{~g}$ four times daily and diclofenac $50 \mathrm{mg}$ twice daily orally were prescribed to all patients for postoperative analgesia. The patients were discharged on the same postoperative day unless otherwise clinically indicated. All patients were asked to clean the wound using a shower spray twice daily. Patients were then followed up in the clinic 2 weeks after discharge and then further reviewed at 3,4 and 6 weeks after surgery.

Pain assessment

Patients were familiarized with an 11-point visual analogue pain score from 0 to 10 . The patients were asked to record at home before bed-time their maximum pain score for the day. The patients were also asked to record the total number of analgesic tablets taken for that day.

Wound healing assessment

The patients' wounds were assessed at scheduled appointments. Wound healing was defined as complete epithelialization as seen on physical examination. The state of the wound was graded according to a five-point scale from sloughy to completely healed (Table 1). A single assessor performed the assessment.

Statistical analysis

Statistical analysis was performed with SPSS software (SPSS for Windows, version 11.5; SPSS, Chicago, IL). The significance of differences was determined using the $t$-test and chi-squared test. Confidence intervals within 95\% were considered significant. Data were stored in a database that was password-secured. A sample size of 90 patients was calculated prior to the study to show a $30 \%$

Table 1 Grades of wound healing

\begin{tabular}{ll}
\hline Grade & Description \\
\hline 1 & Sloughy \\
2 & No granulation \\
3 & Granulating \\
4 & Epithelializing \\
5 & Completely epithelialized \\
\hline
\end{tabular}


difference in wound healing at 3 weeks. This would yield a confidence level of $5 \%$ and a power of $80 \%$.

\section{Results}

The clinical trial was conducted from March 2007 to October 2007. Of 54 patients considered for recruitment. 10 were excluded. Therefore 44 patients were included, and 22 were randomized to LigaSure haemorrhoidectomy and 22 to diathermy haemorrhoidectomy. The flow-chart (Fig. 1) shows the recruitment and randomization process. One patient in the LigaSure arm was lost to follow-up, and thus only 21 patients were evaluated in that group.

The characteristics of the patients in the two arms of the study are shown in Table 2 . There were no statistically significant differences in age, race or sex. The indications for haemorrhoidectomy were similar and both arms had nearly the same percentage of elective operations. The seniority of the surgeon was similar in both arms. Patients in the LigaSure arm had more pile masses removed. The complications in the patients in both arms are shown in
Table 3. There were no statistically significant differences in the incidence of complications between the two groups. The mean operative time for LigaSure haemorrhoidectomy was significantly shorter than that for diathermy haemorrhoidectomy. The time required for LigaSure haemorrhoidectomy was only about half that required for diathermy (Table 4). There was also significantly less intraoperative bleeding during LigaSure haemorrhoidectomy than during diathermy haemorrhoidectomy.

There were no statistically significant differences in the postoperative pain scores between the two groups on days $1,3,7,10$ and 14 after surgery. The mean pain scores were, however, higher in the diathermy group on day 10 and day 14 after surgery. Most patients were painfree by day 21 . There were no statistically significant differences in postoperative analgesic use.

In terms of wound healing, LigaSure haemorrhoidectomy was significantly superior. Of the patients who underwent LigaSure haemorrhoidectomy, 60\% had wounds that were completely epithelialized 3 weeks after surgery compared to $19 \%$ in the diathermy group (Table 4). At 3 weeks after surgery, haemorrhoidectomy per-

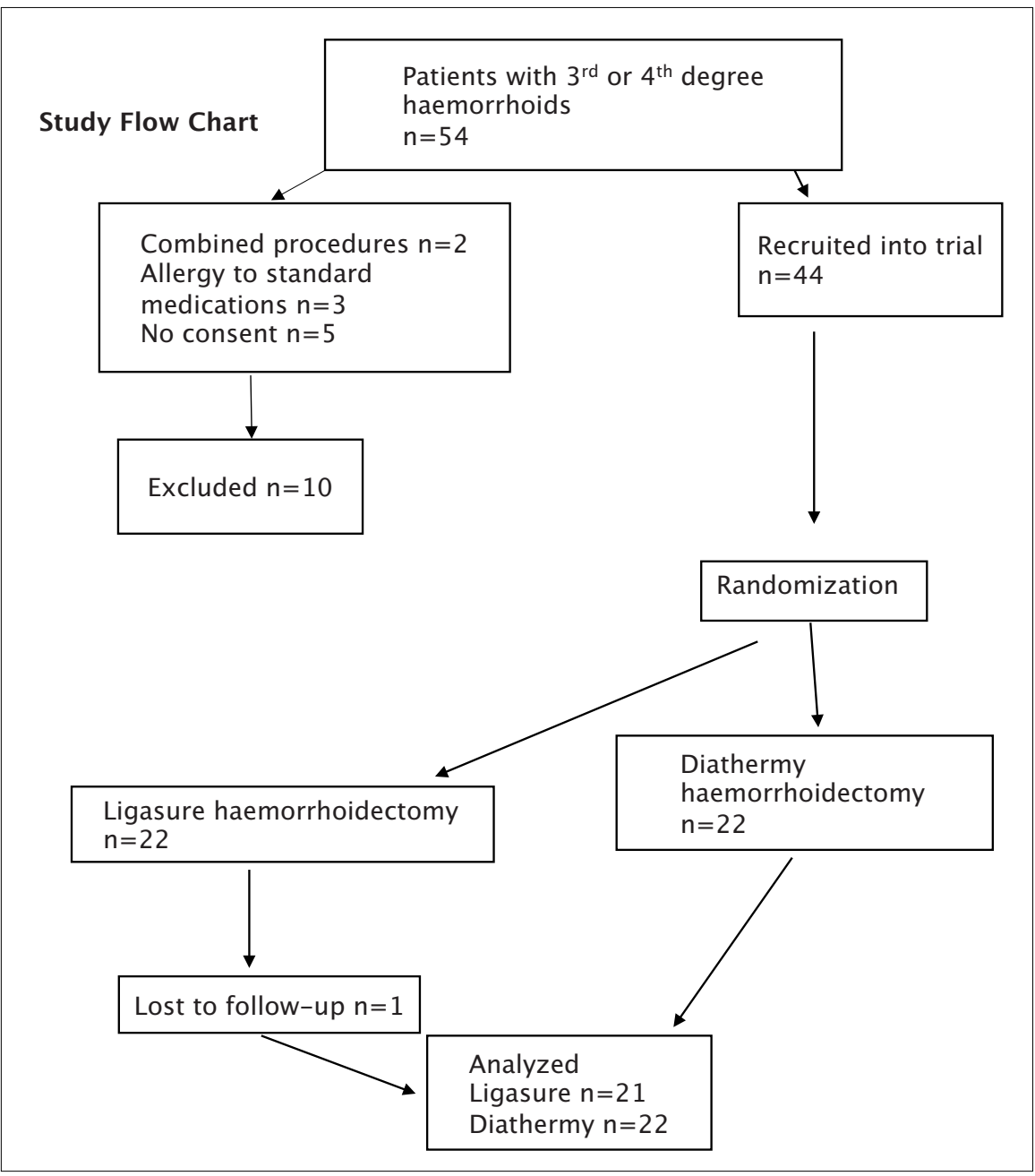

Fig. 1 Study flow chart 
Tables 2 Patient demographics, type of haemorrhoid and surgical aspects in the two treatment groups

\begin{tabular}{|c|c|c|c|}
\hline Variable & LigaSure & Diathermy & $p$ value \\
\hline Mean age (years) & 36.6 & 43.3 & 0.08 \\
\hline Race (\% Chinese) & 90.5 & 72.7 & 0.32 \\
\hline Male sex $(\%)$ & 61.9 & 40.9 & 0.17 \\
\hline Haemorrhoid prolapsed $(\%)$ & 100 & 100 & NS \\
\hline Haemorrhoid thrombosed (\%) & 9.5 & 22.7 & 0.24 \\
\hline Haemorrhoid bleeding (\%) & 95.2 & 72.2 & 0.05 \\
\hline Elective surgery (\%) & 85.7 & 86.3 & 0.95 \\
\hline Mean no. of piles excised & 3.0 & 2.4 & 0.07 \\
\hline Consultant surgeon $(\%)$ & 57.1 & 63.6 & 0.20 \\
\hline
\end{tabular}

Table 3 Complications in the two patient groups

\begin{tabular}{llll}
\hline Complication & LigaSure & Diathermy & $p$ value \\
\hline Pain requiring unscheduled hospital stay (\%) & 9.5 & 9.0 & 0.96 \\
Acute urinary retention (\%) & 0 & 4.5 & 0.32 \\
Delayed bleeding (\%) & 0 & 4.5 & 9.0 \\
Itch (\%) & 9.5 & 0.32 & 0.96 \\
\hline
\end{tabular}

Table 4 Outcomes

\begin{tabular}{|c|c|c|c|}
\hline Outcome & LigaSure & Diathermy & $p$ value \\
\hline Mean operative time (min) & 9.4 & 18.2 & $<0.01$ \\
\hline Mean no. of soaked gauzes & 0.3 & 1.3 & $<0.01$ \\
\hline Wound completely epithelialized by week $3(\%)$ & 60 & 19 & $<0.01$ \\
\hline Wound completely epithelialized by week $4(\%)$ & 95 & 76 & 0.09 \\
\hline
\end{tabular}

formed with LigaSure had an odds ratio for complete epithelialization of 3.1 over diathermy (95\% CI 1.2-8.2). By 4 weeks after surgery $95 \%$ of LigaSure haemorrhoidectomy wounds had healed compared to $76 \%$ in the other group. Nearly all the wounds were completely epithelialized by 6 weeks after surgery.

\section{Discussion}

The LigaSure vessel-sealing system allows complete coagulation of blood vessels up to $7 \mathrm{~mm}$ in diameter while confining the thermal spread to within $2 \mathrm{~mm}$ of adjacent tissue. This advantage has been extended to the excision of haemorrhoids as it allows fast bloodless dissection with minimal collateral damage. Our study confirmed this and showed, as have other studies [5, 6], a significant decrease in operative time and blood loss compared to diathermy. Furthermore, the patients in the LigaSure arm had more pile masses excised (Table 2) than the patients in the diathermy group, suggesting that with the precise dissection possible with LigaSure, better skin bridges can be preserved.

Minimal short-term complications in our patients suggest that LigaSure haemorrhoidectomy is a safe procedure. At 6 weeks follow-up, none of the patients had any signs of anal stenosis. Although anal stenosis has been reported [7], we believe this can be avoided by proper technique and retraction of adjacent perianal skin away from the electrodes. The pile mass also needs to be adequately lifted off the internal sphincter to avoid damage to it.

In contrast to the findings of some similar studies [5, $6]$, we did not find any difference in the pain scores or analgesic use between the LigaSure group and the diathermy group. Anal spasm after haemorrhoidectomy has been implicated in postoperative pain and poor wound healing [8, 9]. It has been postulated that LigaSure haemorrhoidectomy is associated with reduced anal spasm [7]. This is because the collateral damage with LigaSure is less than that with diathermy. We did not see this advantage translate to less pain in patients in the LigaSure group compared to patients in the diathermy group. This may have been because both groups of patients experienced similar inflammatory reaction at the wound thus leading to similar pain scores.

The reduced anal spasm and smaller open wounds associated with LigaSure haemorrhoidectomy may, however, have contributed to earlier wound healing. In a previous trial conducted by us [9], 19\% of patients who underwent diathermy haemorrhoidectomy alone had completely epithelialized wounds at 3 weeks. In this trial, again the complete epithelialization rate at 3 weeks was $19 \%$, illustrating the consistency of our results. The complete epithelialization rate was $60 \%$ at 3 weeks with LigaSure which is extremely encouraging. Although we 
have previously shown that topical glyceryl trinitrate is useful, this was deliberately left out of the current protocol to avoid confounding factors. Combining LigaSure haemorrhoidectomy with topical glyceryl trinitrate may achieve even better wound healing rates.

Although the initially calculated sample size was 90 , the study had to be concluded earlier as the supplier of the LigaSure electrodes could not continue to provide the electrodes for the trial without passing on the cost to the patient. However, as the difference in rate of wound healing was high, a post-hoc power analysis revealed a power of $88.8 \%$ despite the small sample size. We thus contend that the conclusion derived from this study can be considered adequate.

\section{Conclusion}

Although LigaSure haemorrhoidectomy is not associated with less postoperative pain than diathermy, the reduced operative time and bleeding and more rapid wound healing associated with LigaSure haemorrhoidectomy make it superior to diathermy haemorrhoidectomy.

Conflict of interest statement The authors declare that they have no conflict of interest related to the publication of this article.

\section{References}

1. Mattana C, Coco C, Manno A et al (2007) Stapled hemorrhoidopexy and Milligan Morgan hemorrhoidectomy in the cure of fourth-degree hemorrhoids: long-term evaluation and clinical results. Dis Colon Rectum 50:1770-1775

2. Jayaraman S, Colquhoun PH, Malthaner RA (2007) Stapled hemorrhoidopexy is associated with a higher long-term recurrence of internal hemorrhoids compared with conventional excisional hemorrhoid surgery. Dis Colon Rectum 50:1297-1305

3. Milito G, Gargiani M, Cortese F (2002) Randomized trial comparing Ligasure haemorrhoidectomy with the diathermy dissection operation. Tech Coloproctol 6:171-175

4. Jayne DG, Botterill I, Ambrose NS et al (2002) Randomized clinical trial of Ligasure versus conventional diathermy for day-case haemorrhoidectomy. Br J Surg 89:428-432

5. Palazzo FF, Francis DL, Clifton MA (2002) Randomized clinical trial of Ligasure versus open haemorrhoidectomy. Br J Surg 89:154-157

6. Muzi MG, Milito G, Nigro C et al (2007) Randomized clinical trial of LigaSure and conventional diathermy haemorrhoidectomy. Br J Surg 94:937-942

7. Ramcharan KS, Hunt TM (2005) Anal stenosis after LigaSure hemorrhoidectomy. Dis Colon Rectum 48:1670-1671

8. Cheetham MJ, Philips RK (2001) Evidence-based practice in haemorrhoidectomy. Colorectal Dis 3:126-134

9. Tan KY, Sng KK, Eu KW et al (2006) Randomized clinical trial of 0.2 per cent glyceryl trinitrate ointment for wound healing and pain reduction after open diathermy haemorrhoidectomy. Br J Surg 93:1464-1468 